\title{
Comparison between pressure retarded osmosis model using batch and continuous water supply sources
}

\author{
Kingchat Potisa-ad ${ }^{1}$, Lida Simasatitkul $^{2}$, and Suksun Amornraksa ${ }^{1, *}$ \\ ${ }^{1}$ The Sirindhorn International Thai-German Graduate School of Engineering (TGGS), King Monkut's University of Technology North \\ Bangkok, Bangkok, 10800 Thailand \\ ${ }^{2}$ Department of Industrial Chemistry, Faculty of Applied Science, King Monkut's University of Technology North Bangkk, Bangkok, \\ 10800 Thailand
}

\begin{abstract}
Pressure retarded osmosis (PRO) is a novel renewable energy technology that generates electricity from two water sources. Due to the osmotic pressure difference, freshwater permeates across a membrane to the other side, where the high-pressure seawater flows and drives a turbine to generate power. Many mathematic models have been proposed to evaluate the performance of a PRO. However, it was found that most performance of the PRO that have been reported were performance by using freshwater with limited supply (batch) in the model. It is not accurate as, in practice, the supply of freshwater occurs in a continuous manner. In this work, the influence of batch and continuous supply of fresh water on the performance of PRO was demonstrated. The effect of flow direction, i.e., concurrent and counter-current flows, was also examined. The model simulation was performed by using MATLLAB program, and the performance of PRO is expressed in terms of average power density. The results revealed that the batch and continuous supplies of freshwater had a strong impact on the performance of the PRO. However, the performance of concurrent and counter-current flow were not significantly different.
\end{abstract}

Keyword. Pressure retarded osmosis, counter-current flow, Osmotic power

\section{Introduction}

The energy resources for human activities are supported by fossil combustion, which releases high emissions of greenhouse gases. It accelerates climate change and global warming. To reduce the reliance on fossil fuels, alternative sources, which are environmentally friendly, need to be developed and used. Among various renewable energy sources, pressure retarded osmosis is a new energy generation technology that has a good potential to harvest energy from nature. Salinity gradient energy, given by mixing two solutions with different salinity concentrations, is considered promising renewable energy [1]. In theory, the salinity gradient energy has the advantage of high energy density [2]. With a proper method, it is possible to generate electricity from the salinity gradient energy, i.e., by using pressure retarded osmosis (PRO) technology [3].

PRO is an attractive membrane technology for generating electricity by utilization a salinity gradient [4]. This technology operates based on different osmotic pressure between seawater (draw solution) and freshwater (feed solution). Fig. 1 inllustrates a schematic diagram of a typical PRO osmotic pressure process with a continuous and steady-state flow [5]. Both draw and feed solutions are fed into a membrane module with a selected semi-permeable membrane installed to separate both streams. The draw solution is pumped to high pressure then it passes through a pressure exchanger before entering the module, while the feed solution enters the module at atmospheric or low pressure. The osmotic pressure difference between both streams causes the freshwater to permeate across the membrane to the other side where the high salinity seawater flows. Freshwater passing through membrane to salty water increase the volume which is diviede into two streams. The first one is the permeated volume is then used to drive the turbine to produce energy $[5,6]$. Other stream passes through pressure exchanger through pressure exchanger for energy recovery.

The performance of PRO is critical for its economic viability; therefore, it is very important to evaluate the performance accurately and precisely. There are two operation modes of the PRO process; concurrent and counter-current flow operations. Most simulation studies were performed based on the concurrent flow operation, even though some reports indicated that the countercurrent flow is more efficient at extracting energy than concurrent flow [7]. It may be because the simulation steps for concurrent flow are much less complicated than counter-current flow. However, we have noticed that the PRO performance reported in many works of literature was performed based on mathematic models that used a limited supply of freshwater (batch volume) as feed [810]. We realized that they used the batch water supply when we tried to repeat and verify their simulation result. The results from those models that used the batch water supply may not represent the real performance as,

* Corresponding author: suksun.a@tggs.kmutnb.ac.th 
in practice, the actual flow of freshwater is continuous (unlimited supply). Therefore, it is crucial to examine the effect of the batch and continuous freshwater supplies on the performance of PRO. In addition, the draw and feed solutions can flow into the PRO module in two different directions: concurrent and counter-current flows. The different directions may influence the mass transfer between the draw and feed solutions. In this regard, it is also essential to investigate the effect of flow direction on the performance of PRO.

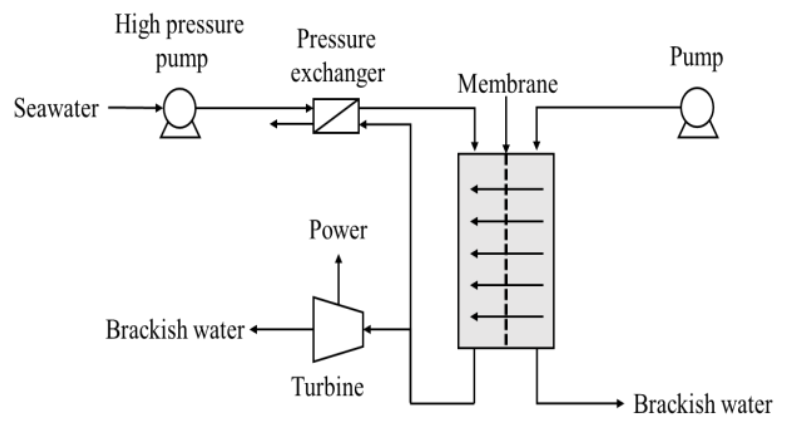

Fig. 1. Pressure retarded osmosis (PRO) presess [5].

\section{Methodology}

A mathematical model for the PRO process was developed and used to calculate the performance of the PRO process. The effect of concentration polarization, which consists of internal concentration polarization (ICP) and external concentration polarization (ECP), was also included in the model. A schematic diagram of the PRO module with concurrent flow and counter-current flow configurations is illustrated in Fig. 2.

(a)

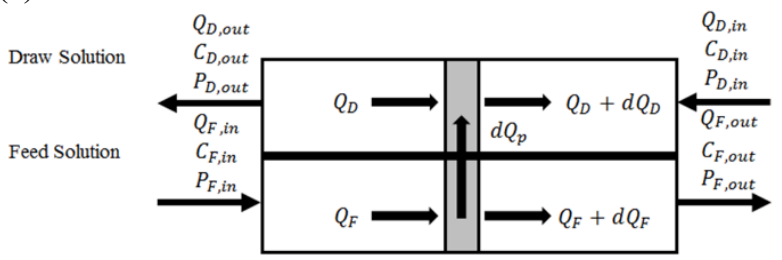

b)

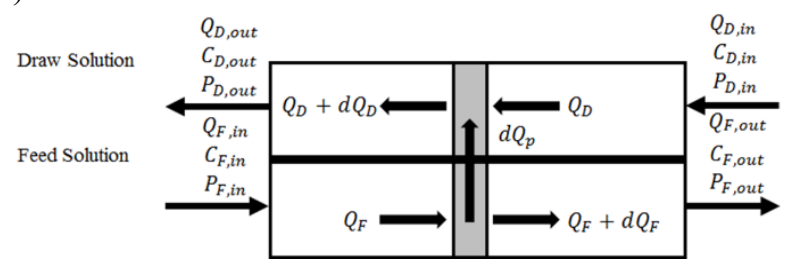

Fig. 2. (a) Schematic diagram of a PRO mass exchanger in concurrent flow configuration. (b) Schematic diagram of a PRO mass exchanger in counter-current flow configuration [11].

A semi-permeable membrane separates the module into two channels. A feed solution with a low salt concentration flows across the membrane to the other side, where a draw solution with high salt concentration flows. Both solutions flow in the same direction for the concurrent flow configuration (Fig. 1a) and in the opposite direction for the counter-current flow configuration (Fig. 1b). The inlet and outlet boundary conditions of both feed and draw streams are given as indicated in Fig. 2.

The performance of the PRO process can be defined as the power density $(\mathrm{W})$, which can be calculated by,

$$
W=A_{W}(\Delta \pi-\Delta P) \Delta P
$$

where $A_{W}$ is the membrane permeability coefficient, $\Delta P$ is the hydraulic pressure difference across the membrane, and $\Delta \pi$ is the osmotic pressure as a function of molar concentration following the van't Hoff equation:

$$
\pi=v R T c
$$

where $v, c, R$, and $T$ are the van't Hoff coefficient, the concentration difference across the membrane, the gas constant, and temperature. The mass transfer equations of water along the membrane channels in concurrent flow module for the draw and feed solution can be expressed as [12]:

$$
\begin{gathered}
\frac{d Q_{D}(S)}{d A_{m}}=J_{W}\left(C_{D}(S), C_{F}(S), \Delta P\right) \\
\frac{d Q_{F}(S)}{d A_{m}}=-J_{W}\left(C_{D}(S), C_{F}(S), \Delta P\right)
\end{gathered}
$$

where $Q_{D}$ and $Q_{F}$ are the flow rates of the draw and feed solutions, respectively, $C_{D}$ and $C_{F}$ are the salt concentrations of the draw and feed solutions, respectively, $A_{W}$ is defined as the total membrane area from the feed entrance, and $J_{W}$ is the water flux through the membrane, which is a function of the salt concentration in the draw and feed solutions and the difference of hydraulic pressure. The salt concentrations distributions $\left(J_{S}\right)$ in both draw and feed channels can be expressed as:

$$
\begin{aligned}
& \frac{d\left(Q_{D}(S)_{C_{D}}(S)\right)}{d A_{m}}=-J_{S}\left(C_{D}(S), C_{F}(S), \Delta P\right) \\
& \frac{d\left(Q_{F}(S)_{C_{D}}(S)\right)}{d A_{m}}=J_{S}\left(C_{D}(S), C_{F}(S), \Delta P\right)
\end{aligned}
$$

In the counter-current flow operation, the mass transfer equations can be expressed as [12]:

$$
\begin{gathered}
\frac{d Q_{D}(S)}{d A_{m}}=J_{W}\left(C_{D}(S), C_{F}(S), \Delta P\right) \\
\frac{d Q_{F}(S)}{d A_{m}}=J_{W}\left(C_{D}(S), C_{F}(S), \Delta P\right) \\
\frac{d\left(Q_{D}(S)_{C_{D}}(S)\right)}{d A_{m}}=-J_{S}\left(C_{D}(S), C_{F}(S), \Delta P\right)
\end{gathered}
$$




$$
\frac{d\left(Q_{F}(S)_{C_{D}}(S)\right)}{d A_{m}}=-J_{S}\left(C_{D}(S), C_{F}(S), \Delta P\right)
$$

With the effect of ICP and ECP, the water permeates flux, and salt permeate flux in PRO modules can be given in equations (11) and (12), respectively [13].

$$
\begin{gathered}
J_{W}=A W\left\{\frac{\pi_{D, b} \exp \left(\frac{-J_{W}}{k}\right)-\pi_{F, b} \exp \left(J_{W} K\right)}{1+\frac{B}{J_{W}}\left[\exp \left(J_{W} K\right)-\exp \left(\frac{-J_{W}}{k}\right)\right]}-\Delta P\right\} \\
J_{S}=B\left\{\frac{\pi_{D, b} \exp \left(\frac{-J_{W}}{k}\right)-\pi_{F, b} \exp \left(J_{W} K\right)}{1+\frac{B}{J_{W}}\left[\exp \left(J_{W} K\right)-\exp \left(\frac{-J_{W}}{k}\right)\right]}-\Delta P\right\}
\end{gathered}
$$

where $\pi_{D, b}$ and $\pi_{F, b}$ are the osmotic pressure of the bulk draw and feed solutions, respectively. $B$ is the solute permeability coefficient, $k$ is the mass transfer coefficient, and $K$ is the solute resistivity for diffusion within the membrane porous support layer, which is calculated from the following equation [14]:

$$
K=\frac{\delta}{D}
$$

In equation (13), $\delta$ is a film thickness on both the feed and draw sides of the membrane, and $D$ is a solute diffusion coefficient. On the other hand, $k$ is calculated by equations (14) and (15), where $d h$ is the hydraulic diameter of the flow channel, $R e$ is Reynolds numbers, $S h$ is the Sherwood number, and $S c$ is the Schmidt number. Due to the mass transfer in the spacer-filled flow channel was turbulent at a relatively low Reynolds number $(\operatorname{Re}<50)$. In this case, the Sherwood number can be determined from equation (15) [15].

$$
\begin{gathered}
k=\frac{S h D}{d h} \\
S h=0.2 \operatorname{Re}^{0.57} S c^{0.4}
\end{gathered}
$$

Other than the power density, the performance of the PRO process is also evaluated in terms of water utilization rate. The water utilization rate is the ratio of the permeate flow rate $\left(Q_{p, w}\right)$ to the inlet flow rate $\left(Q_{F}(\right.$ in $\left.)\right)$ of the feed solution [10] as shown in equations (16) and (17). In implies how efficiently the feed water can be utilized. It is very useful information, especially when the freshwater is limited or not abundant. In the batch supply, the water utilization rate is less than $100 \%$. However, in the continuous supply, the rate can be higher than $100 \%$ as water feed is always replenished along the membrane length.

$$
\% U t=\frac{\sum_{i=1}^{L} Q_{p, w}}{Q_{F(i n)}} \times 100
$$

$$
Q_{p, w}=\int_{a}^{b} J_{W} x d A_{m}
$$

The model simulation in this work was performed by using the MATLAB program. The performance of the PRO process was evaluated in terms of average power density. It is assumed that the pressure drop through the flow channel on both sides of the membrane is negligible, and thus, the hydraulic pressure difference between the draw and feed side remains constant over the membrane length [11]. For simulation, the membrane is divided into $\mathrm{n}$ number of pieces. The water that flows into the channels at the starting point is denoted as $i=0$. The number continuously increases until it reaches $n$ at the ending point. Water flow rates and salt concentration at membrane piece $i+1$ are calculated based on the permeate through membrane piece $\mathrm{i}$. The flow chart for a mathematical model of the PRO power system is summarized in Fig. 3. Note that in the simulation, the feed flow rate decreases along the membrane length in the batch water supply. However, it is constant in the case of continuous supply.

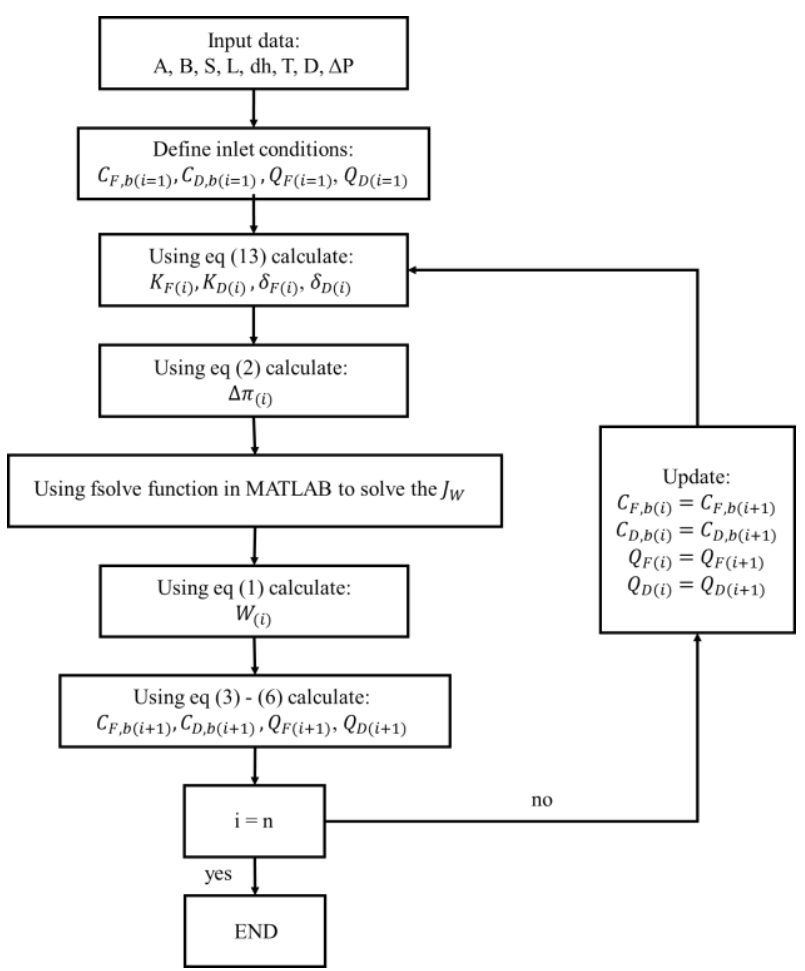

Fig. 3. Flow chart for mathematical model of concurrent PRO power system [8].

\section{Results and discussions}

The effect of batch and continuous water supply sources on the mass transfer of salt and water flux across the membrane were investigated and compared in this section. The configuration and operating conditions used for the simulations in this work were summarized and shown in Table 1. It is important to note that the membrane length at 10 meters was selected to demonstrate the effects more clearly. In practice, the membrane length is usually much shorter. 
Table 1. Conditions for commercial-scale simulation [8].

\begin{tabular}{|l|c|}
\hline \multicolumn{1}{|c|}{ Parameters } & Value \\
\hline Feed concentration, $\mathrm{C}_{\mathrm{F}, \mathrm{b}(\mathrm{in})}(\mathrm{g} / \mathrm{l})$ & 0 \\
\hline Draw concentration, $\mathrm{C}_{\mathrm{D}, \mathrm{b}}(\mathrm{in})(\mathrm{g} / \mathrm{l})$ & 30 \\
\hline Temperature, T ( $\left.{ }^{\circ} \mathrm{C}\right)$ & 25 \\
\hline Membrane length, L (m) & 10 \\
\hline The radius of hollow fiber, $\mathrm{r}(\mathrm{mm})$ & 0.1 \\
\hline Radius of module casing, R (mm) & 0.15 \\
\hline Feed cross-flow velocity, $\mathrm{uF}(\mathrm{in})(\mathrm{m} / \mathrm{s})$ & 0.25 \\
\hline Draw cross-flow velocity, uD (in) (m/s) & 0.25 \\
\hline Salt diffusion coefficient, D (m $\left.{ }^{2} / \mathrm{s}\right)$ & $1.5 \mathrm{e}-9$ \\
\hline
\end{tabular}

\subsection{The effect of concurrent and counter-current flow operation}

First of all, the effect of concurrent and counter-current flow operations was investigated to determine their impact on the PRO performance. The simulation was performed with the continuous water supply power over different applied hydraulic pressures. The power density obtained from both flow directions were illustrated in Fig. 4. It can be seen that the power density obtained from the concurrent and counter-current flow at the same apply hydraulic pressure were very similar. The countercurrent offered slightly better values, especially at the pressure over 10 bars. However, the differences between them are generally less than $5 \%$. Therefore, it can be concluded that the flow direction of the draw and feed solution did not significantly affect the performance of the PRO process. Consequently, to make the simulation much less complicated, all the simulations in the following sections were performed based the concurrent flow operation only.

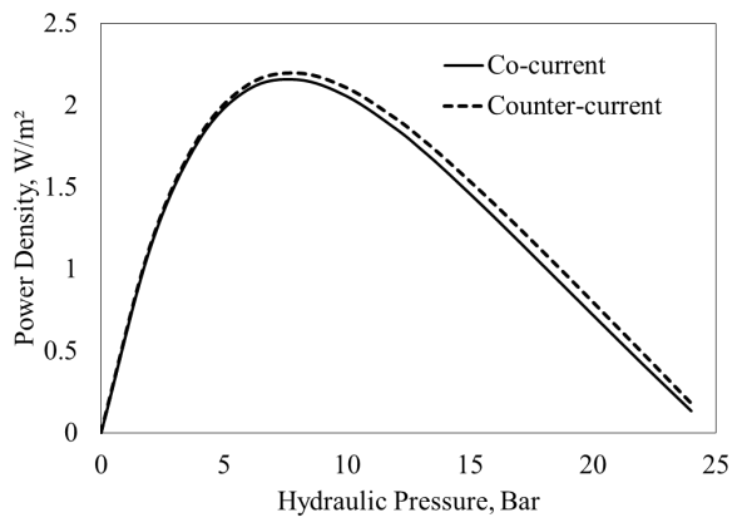

Fig. 4. The power density obtained from the concurrent flow and counter-current flow operations using the continuous supply of fresh water.

\subsection{The effect of batch and continuous water supply sources}

\subsubsection{Salt concentration}

The salt concentration distributions of feed and draw solutions along the membrane length are illustrated in Fig. 5. On the feed solution side, it can be seen that the salt concentration of the continuous feed solution is relatively constant. The reason is simply that the freshwater is continually replenished whenever it permeates across the membrane. So, the overall salt concentration inside the solution did not significantly change. However, on the other hand, the salt concentration of the batch feed solution is increased gradually and significantly along the membrane length. This is because once the freshwater permeates through the membrane, it is gone for good, with no water addition. So, as the salt flux comes from the other side, the salt concentration of the feed solution increases rapidly.

On the draw solution side, it can be seen that the salt concentration gradually decreases along the membrane length as a result of water permeate across the membrane. However, it can be noticed that the salt concentration in the case of batch water supply decreases slowly after passing half of the membrane range. It is likely because the salt concentration in the feed solution is relatively high. It resulted in less driving force for mass transfer (the difference between the osmotic pressure difference and the applied hydraulic pressure). So, lesser and lesser volume of freshwater could permeate across the membrane.

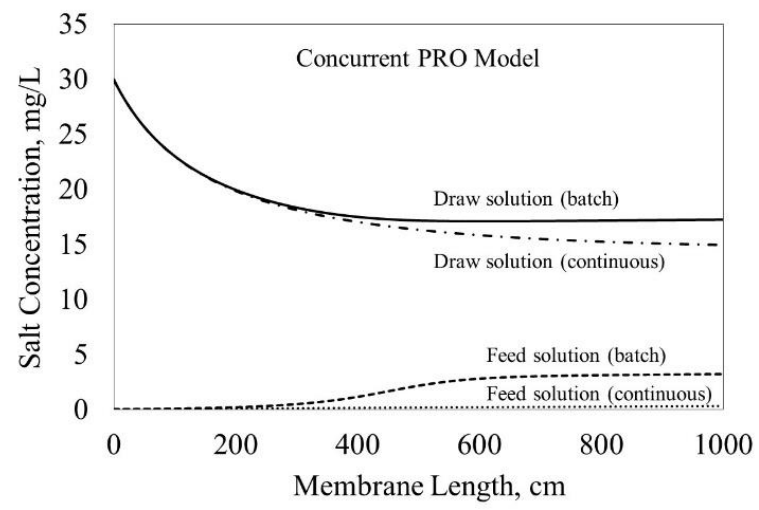

Fig. 5. Salt concentration distribution of draw and feed solutions along the membrane length.

It can be seen that if we operate the PRO process using a very long length of membrane or connecting the membranes in a series, the salt concentration, and thus the osmotic pressure difference, will significantly affect if we select the batch water supply. However, for a short length of membrane, i.e., less than 2 meters, the calculated performance of the PRO may still be acceptable even if the batch water supply is used. 


\subsubsection{Water flux}

The water flux across the membrane length for batch and continuous water supply sources are presented in Fig. 6 . It can be seen from the figure that the water flux for both cases had the same trends, i.e., continuously decreasing along the length of the membrane. However, the water flux of the batch water supply decreased more rapidly than that of the continuous water supply. It happened because there is no new fresh water in the case of the batch water supply; thus, the volume of remaining fresh water on the feed side is continuously decreasing along the membrane length. Consequently, when the salt permeates across the membrane, the salt concentration in the feed solution was increased rapidly. It is different for a continuous water supply because the volume of the remaining fresh water on the feed side is always constant due to fresh water makeup. So, the salt concentration in the feed solution was increased relatively slower than in the batch supply case. As the salt concentration in the feed solution is increased, the driving force, i.e., the osmotic pressure difference between the draw and feed solutions, is also deceased. A simple equation of water flux can explain this. $\mathrm{J}_{\mathrm{W}}=\mathrm{A}(\Delta \pi-\Delta \mathrm{P})$ when the osmotic pressure difference between the draw and feed solutions $\left(\Delta \pi_{\mathrm{D}, \mathrm{b}}-\Delta \pi_{\mathrm{F}, \mathrm{b}}\right)$, which as a function of salt concentration in the draw and feed solutions decreases, the permeate water flux $\mathrm{J}_{\mathrm{W}}$ will be decreased as well. It can be noticed from Fig. 6 that the water flux in batch mode dropped to zero after approximately 6 meters of the length. This result explains why the salt concentration of the draw solution of the batch mode in Fig. 5 no longer decreased after 6 meters.

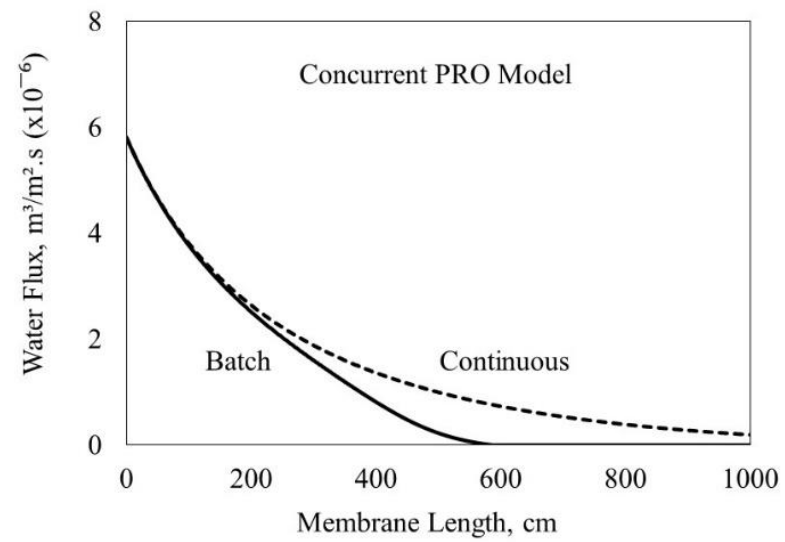

Fig. 6. Comparison of the water flux across the membrane length using batch continuous water supply sources.

In contrast, the water flux in the case of the continuous water supply was continuously penetrated across the membrane until the end of the length, although the salt concentration of the draw solution was continuously decreased. The increase in water flux is directly linked to the power density obtained by the PRO process (eq 1). Hence, it is expected that the power density of the continuous water supply will be higher than the batch water supply.

\subsubsection{Power density}

The power density produced from the PRO using batch and continuous water supply sources at different applied hydraulic pressure was determined and presented in Fig. 7. It can be seen that the power density of the batch and continuous water supply sources were significantly different, especially at the low range of applied hydraulic pressure. However, they are not much different when the applied hydraulic pressure was more than 15 bars. It was found that the maximum power density for the continuous water supply was $2.26 \mathrm{~W} / \mathrm{m}^{2}$ at 8 bars. For the same hydraulic pressure, the power density of the batch water supply as $0.99 \mathrm{~W} / \mathrm{m}^{2}$. Therefore, the percentage of error is 54.2 present. Suppose we use the data from batch water supply to do the economic calculation. Likely, the project will not be viable. Thus, it is essential to do the simulation correctly using the continuous water supply.

In the studies that use freshwater and seawater as the feed and draw solutions, the maximum power density for operation can be achieved when applied hydraulic pressure is equal to half of the osmotic pressure difference $(\Delta \mathrm{P}=\Delta \pi / 2)$ is about 12 bars $[13,8]$. This result is consistent with the result of batch water supply. However, if we use the continuous water supply model, the maximum power density could be obtained at lower applied hydraulic pressures. This result suggests that the PRO process may be suitable to operste at the applied hydraulic pressures lower than half of the osmotic pressure difference. This lower pressure condition is favorable as it can reduce the electricity cost of the pump and give more flexibility for the design and construction of the PRO system.

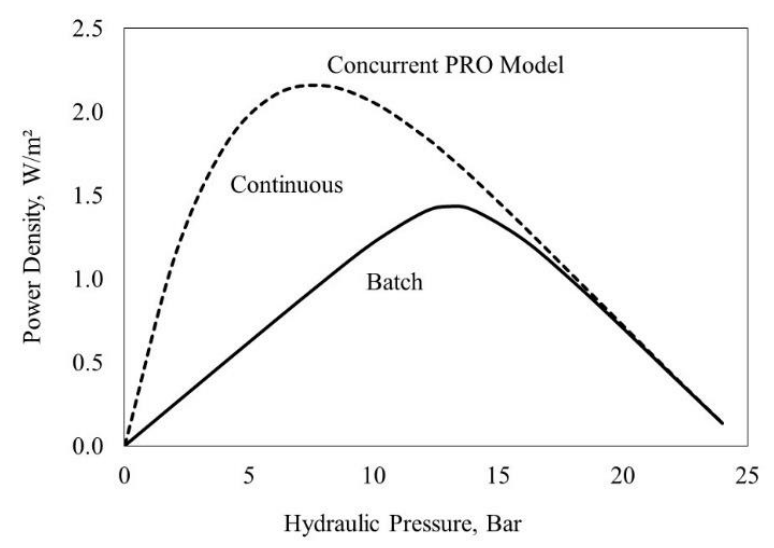

Fig. 7. Comparison of the power density of PRO obtained from batch and continuous water supply sources

\subsubsection{Water utilization}

The water utilization obtained from the batch and continuous water supply sources at different applied hydraulic pressure were calculated and shown in Fig. 8. In general, it can be seen that the maximum water utilization for batch water supply is 100 percent as all the water permeates through the membrane, and no additional water was added. On the other hand, at high pressure, the water hardly permeated through the 
membrane. However, some water remained in the water solution feed, and thus the water utilization was not 100 percent.

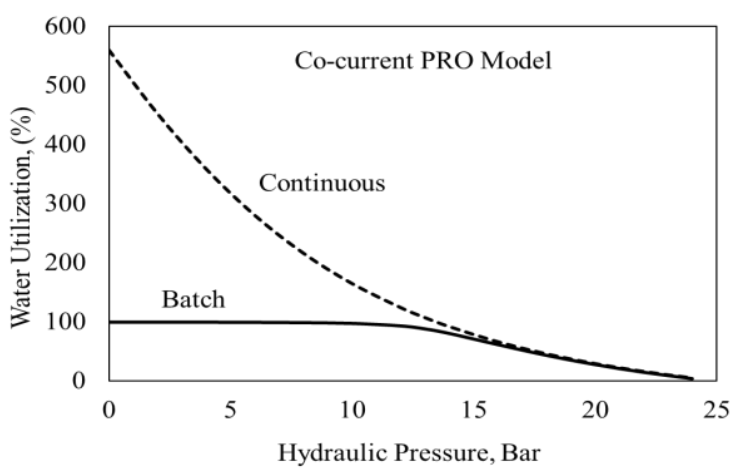

Fig. 8. Comparison of the water utilization of PRO obtained from batch and continuous water supply sources

In the continuous water supply, the water could easily permeate through the membrane, especially at low applied hydraulic pressure. As the water supply was constantly replenished, more volumes of water could permeate through the membrane. It made the percentage of water utilization to be higher than $100 \%$. So, it depends on the availability of freshwater. We could formulate an operating strategy that utilizes the freshwater supply most efficiently while maximizing the power density.

\section{Conclusion}

For the results, it can be concluded that the effect of flow direction, i.e., concurrent and counter-current, did not significantly affect the performance of the PRO. On the other hand, the batch and continuous water supply sources have a strong influence on the PRO's performance. Using the continuous water supply can increase permeate water through the membrane, boosting the power density by $54.2 \%$. Therefore, it is very important to select the condition of the water supply for the simulation carefully. Unless the designers have a specific reason to choose the batch operation, the continuous water supply should be used to simulate and estimate the project's cost.

\section{Acknowledgement}

This work was supported from King Mongkut's University of Technology North Bangkok found (KMUTNB-60-GEN-050) is gratefully acknowledged.

\section{References}

1. B. E. Logan, M. Elimelech, Membrane-based processes for sustainable power generation using water, nature, 488 (2012): 313-319.
2. N. Y. Yip, M. Elimelech, Thermodynamic and energy efficiency analysis of power generation from natural salinity gradients by pressure retarded osmosis, Environmental Science \& Technology, 46 (2012): 5230-5239.

3. A.G.L. Borthwick, Marine renewable energy seascape, Engineering, 2 (2016): 69-78.

4. S. Loeb, R.S. Norman, Osmosis power plants, Science, 189 (1975): 654-655.

5. G. Han, S. Zhang, X. Li, and T.S. Chung, Progress in pressure retarded osmosis (PRO) membrane for osmotic power generation, Progress in polymer science, 51 (2015): 1-27.

6. S. Loeb, Large-scale power production by pressureretarded osmosis using river water and seawater passing through spiral modules, Desalination, 143 (2002): 115-122.

7. S. Lin, A.P. Straub, M. Elimelech, Thermodynamic limits of extractable energy by pressure retarded osmosis, Energy \& Environmental Science, 7 (2014): 2706-2714.

8. M. F. Naguib, J. Maisonneuve, C.B. Laflamme, and P. Pillay, Modeling pressure-retarded osmotic power in commercial length membrane, Renewable energy, 76 (2015): 619-627.

9. J. Maisonneuve, P. Pillay, C.B. Laflamme, Pressureretarded osmotic power system model considering non-ideal effects, Renewable energy, 75 (2015): 416-424.

10. W. Yang, L. Song, J. Zhao, Y. Chen, and B. Hu, Numerical analysis of performance of ideal countercurrent flow pressure retarded osmosis, Desalination, 433 (2018): 41-472.

11. M. H. Sharqawy, and L. D. Banchik, Effectivenessmass transfer unit (E-MUT) model of an ideal pressure retarded osmosis membrane mass exchanger, Journal of membrane science, 445 (2013): 211-219.

12. A. P. Straub, S. Lin, and M. Elimelech, Modulescale analysis of pressure retarded osmosis: performance limitations and implications for fullscale operation, Environmental science \& Technology, 445 (2013): 211-219.

13. A. Altaee, and N. Hilal, Dual stage PRO power generation from brackish water brine and wastewater effluent feeds, Desalination, 389 (2016): 68-77.

14. A. Achilli, T.Y. Cath, and A.E. Childress, Power generation with pressure retarded osmosis: an experimental and theoretical investigation, Journal of membrane science, 343 (2009): 42-52.

15. C.P. Koutsou, S.G. Yiantsios, A.J. Karabelas, A numerical and experimental study of mass transfer in spacer-filled channels: Effects of spacer geometrical characteristics and Schmidt number, Journal of Membrane Science, 326 (2009): 234-251. 